\title{
Epithelial-Mesenchymal Transition Phenotype, Metformin, and Survival for Colorectal Cancer Patients with Diabetes Mellitus II
}

\author{
Yaodu Wang, ${ }^{1}$ Zhiyang $\mathrm{Wu}^{2}$ and Likuan $\mathrm{Hu}^{1}$ \\ ${ }^{1}$ Cancer Center, Shandong University Qilu Hospital, West Wenhua Road 107, Jinan, Shandong Province 250012, China \\ ${ }^{2}$ Intensive Care Unit, Shandong University Qilu Hospital (Qingdao), Hefei Road 758, Qingdao, Shandong Province 266035, China \\ Correspondence should be addressed to Likuan Hu; likuanhu@163.com
}

Received 14 March 2017; Revised 26 May 2017; Accepted 30 May 2017; Published 28 June 2017

Academic Editor: Joanne Bowen

\begin{abstract}
Copyright (c) 2017 Yaodu Wang et al. This is an open access article distributed under the Creative Commons Attribution License, which permits unrestricted use, distribution, and reproduction in any medium, provided the original work is properly cited.
\end{abstract}

\begin{abstract}
Objectives. We aimed to explore the association between metformin treatment and epithelial-mesenchymal transition (EMT) phenotype and further appraise the prognostic values of metformin and EMT markers E-cadherin and vimentin for colorectal cancer (CRC) in clinical practice. Methods. We collected specimens and evaluated clinicopathological parameters of 102 stage I to III CRC patients with prediagnosed type 2 diabetes mellitus (DM II). Expression of E-cadherin and vimentin in tumors was detected by immunohistochemistry (IHC), and statistical analysis was performed using SPSS 19.0. Results. In correlation tests, we found a lower tumor cell EMT degree (more E-cadherin $(P=0.014)$ and less vimentin $(P=0.011)$ expression in patients who used metformin, and the expression of E-cadherin and vimentin was associated with serum CA19-9 $(P=0.048$, $P=0.009)$, tumor invasive depth $(\mathrm{T})(P<0.001, P=0.045)$, and lymph invasion $(\mathrm{N})(P=0.013, P=0.001)$. In Cox multivariate regression analysis, E-cadherin was identified as a prognostic factor for disease-free survival $(\mathrm{DFS})(P=0.038)$ and metformin use $(P=0.015 P=0.044)$ and lymph invasion $(P=0.016 P=0.023)$ were considered as the prognostic factors for both DFS and overall survival (OS). Conclusion. Our study suggested that metformin may impede the EMT process and improve survival for stage I-III CRC patients with DM II.
\end{abstract}

\section{Introduction}

Colorectal cancer (CRC) is the third most common cancer and the fourth leading cause of cancer-related death worldwide [1]. With proper treatments, CRC patients in the early stages have been able to achieve long diseasefree survival (DFS). Several studies have indicated that CRC and many other cancer patients with diabetes mellitus (DM) tend to have a worse prognosis than patients without DM [2-5]. Over the last decade, metformin, as a classical oral hypoglycemic agent, has been discovered to possess antineoplastic activities $[6,7]$ and has been proven effective in survival improvement for CRC patients with type II DM (DM II) [8-12].

Epithelial-mesenchymal transition (EMT) is a process by which epithelial cells lose epithelial characteristics, such as polarity and adhesion, gain migratory properties, and transform into mesenchymal cells [13]. EMT is widely occurring in embryonic development, tissue regeneration, fibrosis, and cancer [14]. EMT has been found to be associated with invasion, metastasis, recurrence, and drug resistance in cancer $[15,16]$. Based on a series of antineoplastic evidence from previous reports, the anti-EMT activity of metformin was also tested in a few studies. With metformin exposure, EMT inhibition was observed in breast cancer, melanoma, prostate cancer, lung cancer, and thyroid cancer cells [17-24]. However, all the abovementioned anti-EMT studies were performed using cell lines in vitro, and there is still little analogous research for CRC EMT.

Here, we aim to explore the association between metformin use and EMT degree for CRC patients with DM II in a clinical setting. In our study, clinicopathologic parameters were evaluated and the expression of EMT markers E- 
cadherin and vimentin in tumor tissue was detected by immunohistochemistry (IHC). Conclusions were based on the outcomes from statistical analysis including correlation tests and survival modeling for variables.

\section{Materials and Methods}

2.1. Patients. Patients with DM II, diagnosed pathologically with stage I to III colorectal cancer who underwent radical surgery in Qilu Hospital of Shandong University between 2008 and 2012, were eligible for our study. The clinical parameters of patients were obtained from the electronic medical record (EMR) system and case follow-ups. The exclusion criteria were set as follows: (1) secondary primary malignant tumor; (2) inflammatory bowel disease (IBD) or Peutz-Jeghers syndrome; (3) diagnosed with DM II less than 1 year before tumor diagnosis [25]; (4) administered metformin less than 6 months before tumor diagnosis [2]; (5) pathologic type other than adenocarcinoma; (6) accepted anticancer treatment before surgery; (7) did not reach R0 resection; (8) and incomplete medical records. The primary tumor was evaluated by the tumor-nodemetastasis (TNM) staging system (American Joint Committee on Cancer (AJCC), version 7.0), and therapy outcome was assessed by the Response Evaluation Criteria in Solid Tumor (RECIST, version 1.1).

2.2. Specimen and IHC. The paraffin-embedded tumor tissues from radical surgery were cut into slices. After dewaxing and dehydrating, slides were subjected to antigen retrieval by citric acid treatment $(\mathrm{pH}$ 6.0) and microwave. Slides were then treated with $\mathrm{H}_{2} \mathrm{O}_{2}$ to block endogenous peroxidase activity and subsequently incubated with $5 \%$ bovine serum albumin phosphate-buffered saline (BSA-PBS) solution, diluted monoclonal rabbit anti-E-cadherin (Cell Signaling Technology, USA), and antivimentin antibody (Cell Signaling Technology, USA) at $4^{\circ} \mathrm{C}$ overnight. The poly-horseradish peroxidase (Poly-HRP) immunoglobulin G (IgG) detection kit (ZSGB-BIO, China P. R.) and DAB kit (ZSGB-BIO, China P. R.) were used for detection and staining. The DAB-stained slides were counterstained with hematoxylin, dehydrated with alcohol, and cleared with xylene. Slides of normal colon and rhabdomyosarcoma tissue were also tested for the positive controls of E-cadherin and vimentin, respectively. BSA-PBS solution (5\%) without antibody was used for negative controls.

The stained slides were scored under a light microscope by 3 pathologists according to the following criteria: (1) intensity of staining: 1 = weak staining, 2 =moderate staining, and $3=$ strong staining; (2) percentage of stained cells: $1=1-10 \%, 2=11-35 \%, 3=36-65 \%$, and $4=66-100 \%$. The total score was calculated by the sum of intensity and percentage scores, and a total score $>3$ was defined as positive expression. Pathologists participating in scoring were blinded to clinical data.

2.3. Statistical Analysis. The primary endpoint of the study was overall survival (OS). Student's $t$-test was used for continuous variable analysis, and Pearson's chi-squared $\left(\chi^{2}\right)$ test was used for comparison of categorical variables. Survival data were analyzed using the Kaplan-Meier method and compared by log-rank statistics. Cox regression was used for multivariate survival analysis. Outcomes with a $P<0.05$ were considered significant. All statistical analyses were performed with SPSS 19.0 software.

2.4. Ethics Statement. This study was approved by the Ethics Committee of Qilu Hospital, and all patients provided informed consent.

\section{Results}

3.1. Patient Characteristics. A final total of 102 of 115 patients were included in the study (7 patients were excluded because the DM II diagnosis date was less than 6 months before surgery, 2 patients were excluded for receiving presurgery chemotherapy, and 4 patients were lost to follow-up). The median duration of follow-up was 1678 days. Clinicopathologic parameters of the cohort, such as age, gender, smoking and drinking history, family history, tumor location, staging, invasive depth, lymph invasion, and E-cadherin and vimentin expression are shown in Table 1. Of the total patients, $28(27.45 \%)$ were metformin users, 49 (48.04\%) were detected as positive for E-cadherin expression, and 55 (53.92\%) were detected positive for vimentin expression (representative pictures in Figure 1).

3.2. Association between Metformin Use, E-cadherin and Vimentin Expression, and Clinicopathologic Parameters. As presented in Table 1 , insulin injection $(P=0.018)$ and $E-$ cadherin $(P=0.014)$ and vimentin $(P=0.011)$ expression were significantly associated with metformin use. Using the Mann-Whitney $U$ test for IHC scores (Figure 2), we further observed trends that patients using metformin expressed higher levels of E-cadherin $(P<0.001)$ and lower levels of vimentin $(P=0.001)$ than those without metformin use. Patients with metformin treatment showed less lymph invasion $(P=0.041)$. Significant relationships were not observed with gender, age, body mass index (BMI), tumor markers, and other variables except family malignant history $(P=0.016)$.

In Table 2, expression of both E-cadherin and vimentin showed significant associations with tumor invasive depth $(\mathrm{T})(P<0.001, P=0.045)$ and lymph invasion $(\mathrm{N})$ $(P=0.013, P=0.001)$. As presurgery tumor markers, Ecadherin was correlated with both serum CEA $(P=0.007)$ and CA19-9 $(P=0.048)$ and vimentin was correlated with serum CA19-9 $(P=0.009)$. No significant associations were observed for tumor site, histologic grade, sulfonylurea use, or other parameters.

3.3. Survival Analysis. Recurrence was diagnosed in 46 (45.01\%) patients and death occurred in 37 (36.27\%) patients by the end of follow-up.

Univariate survival analysis using the Kaplan-Meier method (Table 3 ) showed that normal presurgery serum CA19-9 values $(P=0.006, P=0.005)$, metformin use $(P=0.003, \quad P=0.004)$, shallow tumor invasive depth (T1-3) $\quad(P=0.004, \quad P=0.010)$, less lymph invasion 
TABLE 1: Clinicopathologic characteristics of 102 patients.

\begin{tabular}{|c|c|c|c|c|}
\hline Variable & Category & Metformin group & Nonmetformin group & $P$ \\
\hline Age & & 63.57, mean & 63.74, mean & 0.596 \\
\hline \multirow{2}{*}{ Gender } & Female & 10 & 25 & 0.855 \\
\hline & Male & 18 & 49 & \\
\hline \multirow{2}{*}{ Drinking history } & Yes & 8 & 12 & 0.161 \\
\hline & No & 20 & 62 & \\
\hline \multirow{2}{*}{ Smoking history } & Yes & 7 & 23 & 0.547 \\
\hline & No & 21 & 51 & \\
\hline \multirow{2}{*}{ Family history } & Yes & 5 & 2 & $0.016^{*}$ \\
\hline & No & 23 & 72 & \\
\hline BMI & & 25.46 , mean & 24.73 , mean & 0.889 \\
\hline CEA & & 5.68, mean & 4.11, mean & 0.228 \\
\hline CA19-9 & & 9.7 , mean & 11.97, mean & 0.172 \\
\hline \multirow{2}{*}{ Sulfonylurea } & Yes & 13 & 34 & 0.965 \\
\hline & No & 15 & 40 & \\
\hline \multirow{2}{*}{ Insulin } & Yes & 4 & 30 & $0.018^{*}$ \\
\hline & No & 24 & 44 & \\
\hline \multirow{5}{*}{ Site } & Right & 7 & 12 & 0.787 \\
\hline & Transverse & 1 & 4 & \\
\hline & Left & 1 & 5 & \\
\hline & Sigmoid & 6 & 13 & \\
\hline & Rectum & 13 & 40 & \\
\hline \multirow{2}{*}{ Histologic grade } & I-II & 22 & 63 & 0.427 \\
\hline & III & 6 & 11 & \\
\hline \multirow{2}{*}{$\mathrm{T}$} & T1-3 & 18 & 34 & 0.098 \\
\hline & $\mathrm{T} 4$ & 10 & 40 & \\
\hline \multirow{2}{*}{$\mathrm{N}$} & N0 & 21 & 39 & $0.041^{*}$ \\
\hline & N1-2 & 7 & 35 & \\
\hline \multirow{2}{*}{ E-cadherin } & Positive & 19 & 30 & $0.014^{*}$ \\
\hline & Negative & 9 & 44 & \\
\hline \multirow{2}{*}{ Vimentin } & Positive & 8 & 42 & $0.011^{*}$ \\
\hline & Negative & 20 & 32 & \\
\hline
\end{tabular}

${ }^{*} P<0.05$

(N) $(P<0.001, \quad P<0.001)$, positive E-cadherin expression $(P=0.001, \quad P=0.003)$, and negative vimentin expression $(P=0.019, P=0.012)$ contributed to both longer DFS and OS. Alcohol drinking, smoking, family history, serum CEA level presurgery, sulfonylurea or insulin use, tumor histologic grade, and adjuvant chemotherapy were not associated with survival.

The prognostic values of variables were further tested by multivariate Cox regression (Table 4). Metformin use $(P=0.015, \quad P=0.044)$ and lymph invasion $(P=0.016$, $P=0.023)$ were confirmed as significant independent prognostic factors for both DFS and OS. E-cadherin expression $(P=0.038)$ was considered to be a prognostic factor only for DFS. Alcohol drinking $(P=0.035)$ was determined to be a risk factor for DFS, whereas tumor invasive depth $(P=0.066, P=0.108)$ and vimentin expression $(P=0.369, P=0.900)$, which were significant in
Kaplan-Meier analysis, were not identified as prognostic factors in the multivariate model.

\section{Discussion}

Our study explored the association between metformin use and EMT marker (E-cadherin and vimentin) expression in 102 CRC patients with DM II and further estimated the prognostic values of metformin, EMT markers, and other clinicopathologic parameters.

As common molecular markers for EMT studies, Ecadherin performs cell to cell adhesive functions for the epithelium and is conventionally used to measure cell epithelization [26]; vimentin is a major cytoskeletal component for mesenchymal cells and is routinely applied in identifying mesenchyme [27]. Correlation analysis in our study revealed the expression discrepancy of E-cadherin and vimentin 


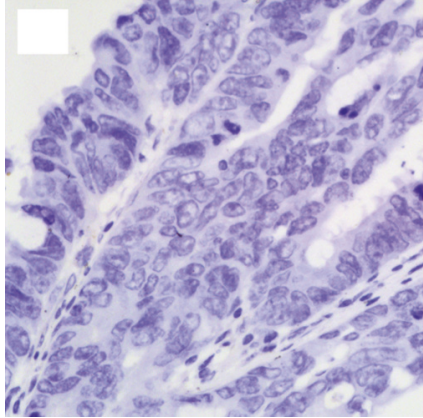

(a)

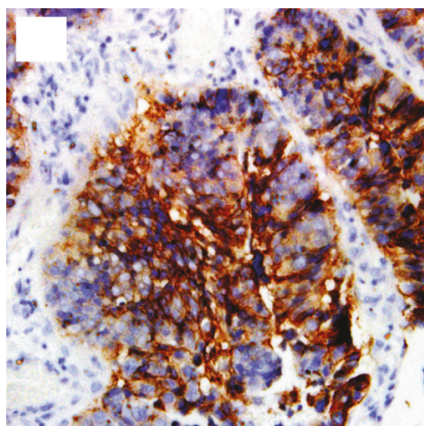

(c)

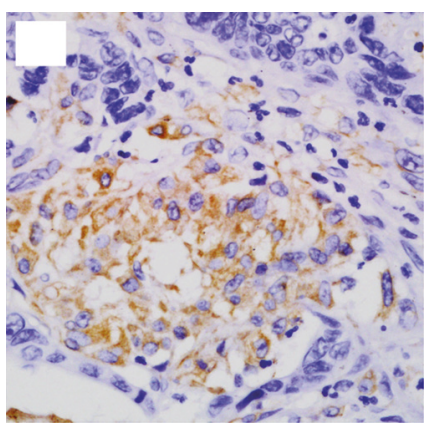

(e)

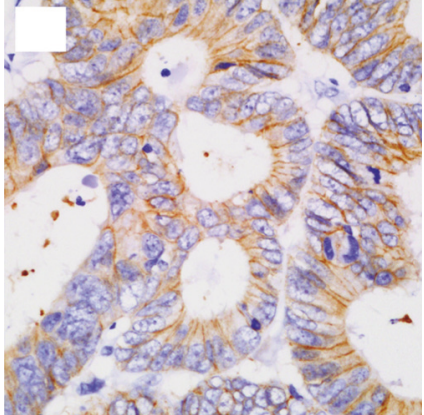

(b)

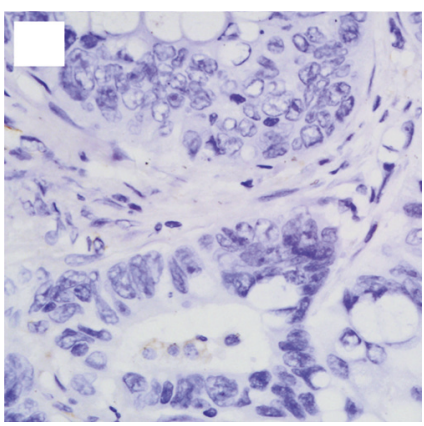

(d)

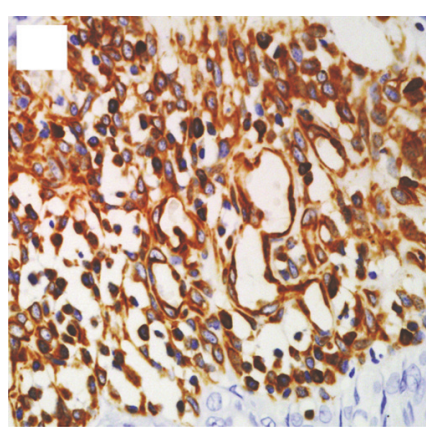

(f)

FIGURE 1: Representative pictures by immunohistochemistry for E-cadherin and vimentin expression in specimen. (Magnification $\times 400$ ) E-cadherin expression: (a) negative expression, (b) low expression, and (c) high expression. Vimentin expression: (d) negative expression, (e) low expression, and (f) high expression.

between the metformin group and the nonmetformin group: patients in the metformin group showed higher E-cadherin and lower vimentin expression in tumors, indicating lower proportions of EMT cells and lower EMT degree of malignant tissues. The results suggested that metformin use may impede the EMT process of CRC.

Among numerous studies on metformin's antiplastic mechanisms, EMT inhibition was first reported in MDAMB-231 and transforming growth factor beta- (TGF- $\beta$-) induced MCF-7 breast cancer cells $[17,18]$. This effect was discovered with the regulation of the cancer stem cell phenotype and has been further verified in other cell lines by previous studies [19-24]. To the best of our knowledge, this is the first study to reveal the anti-EMT potential of metformin in CRC. Although analogous results had not been directly reported in previous laboratory studies, the following clues were adequate to support our conclusion.
According to existing studies, the main mechanism of metformin action in DM II treatment is AMP-activated protein kinase (AMPK) activation [28]. By activating AMPK and downstream messengers, metformin suppresses gluconeogenesis, downregulates blood sugar and insulin levels, and moderates the metabolism of glucose and fat [29]. Similarly, in the anticancer studies, researchers have found that metformin can impede tumor growth by activating the AMPK pathway [30, 31]. For human colorectal cell lines HT29 and drug-resistant cell line LoVo, metformin was observed to activate AMPK and inhibit cell growth [32,33]. Additionally, AMPK pathway activation was considered as an alternative approach to inhibit EMT [34], and metformin has also been proven to perform such inhibition through AMPK activation for melanoma and breast cancer cells $[19,20]$.

Researchers have also confirmed that several other signaling pathways such as TGF- $\beta$, JAK-STAT, PI3K/Akt/ 

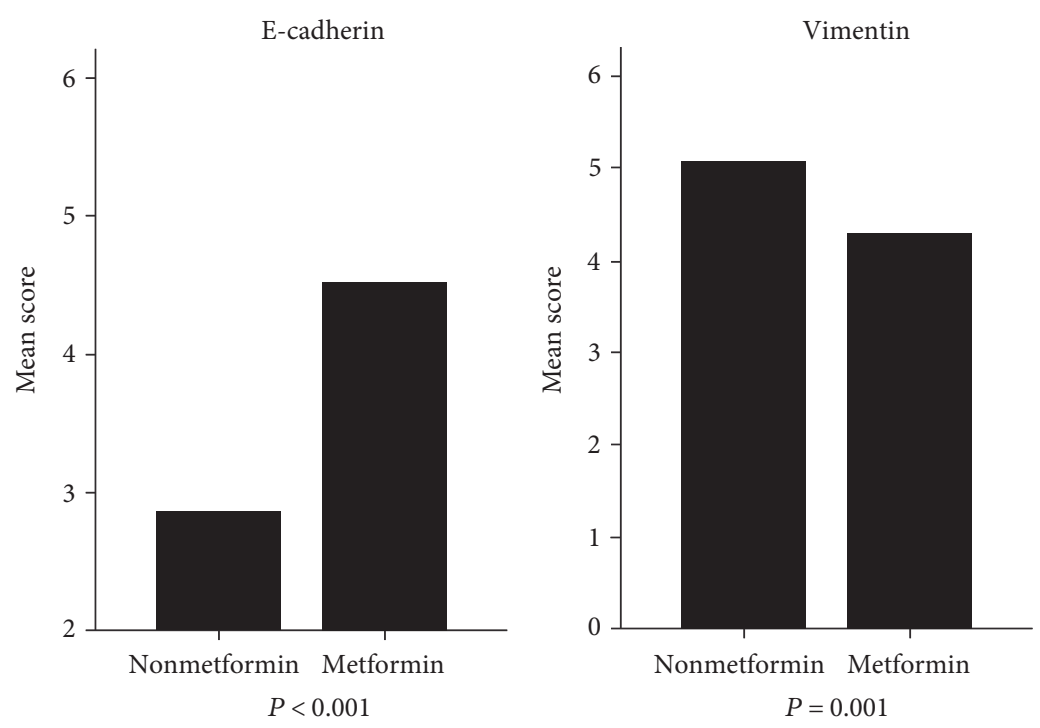

FIGURE 2: IHC scores of E-cadherin and vimentin in CRC tissues from patients with or without metformin: compared with patients without metformin, patients with metformin expressed higher E-cadherin $(P<0.001)$ and lower vimentin $(P=0.001)$.

TABle 2: Associations between expression of E-cadherin, vimentin, and clinicopathologic characteristics.

\begin{tabular}{|c|c|c|c|c|c|c|c|}
\hline \multirow{2}{*}{ Variable } & \multirow{2}{*}{ Category } & \multicolumn{2}{|c|}{ E-cadherin } & \multirow{2}{*}{$P$} & \multicolumn{2}{|c|}{ Vimentin } & \multirow{2}{*}{$P$} \\
\hline & & Positive & Negative & & Positive & Negative & \\
\hline Age & & 64.24 , mean & 63.19 , mean & 0.639 & 64.62, mean & 62.69 , mean & 0.501 \\
\hline \multirow{2}{*}{ Gender } & Female & 15 & 20 & 0.449 & 18 & 17 & 0.715 \\
\hline & Male & 34 & 33 & & 37 & 30 & \\
\hline \multirow{2}{*}{ Drinking history } & Yes & 9 & 11 & 0.762 & 7 & 13 & 0.058 \\
\hline & No & 40 & 42 & & 48 & 34 & \\
\hline \multirow{2}{*}{ Smoking history } & Yes & 16 & 14 & 0.490 & 16 & 14 & 0.939 \\
\hline & No & 33 & 39 & & 39 & 33 & \\
\hline \multirow{2}{*}{ Family history } & Yes & 4 & 3 & 0.708 & 3 & 4 & 0.543 \\
\hline & No & 45 & 50 & & 52 & 43 & \\
\hline BMI & & 25.04 , mean & 24.83 , mean & 0.876 & 24.68, mean & 25.2 , mean & 0.888 \\
\hline CEA & & 8.24 , mean & 14.23 , mean & $0.007^{*}$ & 13.76, mean & 8.75, mean & 0.105 \\
\hline CA19-9 & & 23.46, mean & 36.58 , mean & $0.048^{*}$ & 42.27, mean & 17.31, mean & $0.009^{*}$ \\
\hline \multirow{2}{*}{ Sulfonylurea } & Yes & 27 & 20 & 0.079 & 24 & 23 & 0.592 \\
\hline & No & 22 & 33 & & 31 & 24 & \\
\hline \multirow{2}{*}{ Insulin } & Yes & 13 & 21 & 0.161 & 24 & 11 & $0.032^{*}$ \\
\hline & No & 36 & 32 & & 31 & 36 & \\
\hline \multirow{5}{*}{ Site } & Right & 9 & 10 & 0.934 & 9 & 10 & 0.770 \\
\hline & Transverse & 2 & 3 & & 2 & 3 & \\
\hline & Left & 2 & 4 & & 4 & 2 & \\
\hline & Sigmoid & 10 & 9 & & 9 & 10 & \\
\hline & Rectum & 26 & 27 & & 31 & 22 & \\
\hline \multirow{2}{*}{ Histologic grade } & I-II & 40 & 45 & 0.658 & 43 & 42 & 0.131 \\
\hline & III & 9 & 8 & & 12 & 5 & \\
\hline \multirow{2}{*}{$\mathrm{T}$} & $\mathrm{T} 1-3$ & 35 & 17 & $<0.001^{*}$ & 23 & 29 & $0.045^{*}$ \\
\hline & $\mathrm{T} 4$ & 14 & 36 & & 32 & 18 & \\
\hline \multirow{2}{*}{$\mathrm{N}$} & N0 & 35 & 25 & $0.013^{*}$ & 24 & 36 & $0.001^{*}$ \\
\hline & N1-2 & 14 & 28 & & 31 & 11 & \\
\hline
\end{tabular}

${ }^{*} P<0.05$. 
TABLE 3: Kaplan-Meier analysis for disease-free survival (DFS) and overall survival (OS).

\begin{tabular}{|c|c|c|c|c|c|c|c|}
\hline \multirow{2}{*}{ Variable } & \multirow{2}{*}{ Category } & \multicolumn{2}{|c|}{ Recurrence } & \multirow{2}{*}{$P$} & \multicolumn{2}{|c|}{ Death } & \multirow{2}{*}{$P$} \\
\hline & & Yes & No & & Yes & No & \\
\hline \multirow{2}{*}{ Age (year) } & $\leq 50$ & 7 & 8 & 0.942 & 6 & 9 & 0.758 \\
\hline & $>50$ & 39 & 48 & & 31 & 56 & \\
\hline \multirow{2}{*}{ Gender } & Female & 14 & 21 & 0.775 & 11 & 24 & 0.562 \\
\hline & Male & 32 & 35 & & 26 & 41 & \\
\hline \multirow{2}{*}{ Drinking history } & Yes & 5 & 15 & 0.080 & 4 & 16 & 0.098 \\
\hline & No & 41 & 41 & & 33 & 49 & \\
\hline \multirow{2}{*}{ Smoking history } & Yes & 12 & 18 & 0.324 & 9 & 21 & 0.152 \\
\hline & No & 34 & 38 & & 28 & 44 & \\
\hline \multirow{2}{*}{ Family history } & Yes & 3 & 4 & 0.786 & 3 & 4 & 0.973 \\
\hline & No & 43 & 52 & & 34 & 61 & \\
\hline \multirow{2}{*}{ BMI $\left(\mathrm{kg} / \mathrm{m}^{\wedge} 2\right)$} & $\leq 25$ & 22 & 31 & 0.633 & 20 & 33 & 0.598 \\
\hline & $>25$ & 24 & 25 & & 17 & 32 & \\
\hline \multirow{2}{*}{ CEA (ng/ml) } & $\leq 5$ & 23 & 35 & 0.279 & 18 & 39 & 0.650 \\
\hline & $>5$ & 23 & 21 & & 19 & 26 & \\
\hline \multirow{2}{*}{ CA19-9 (U/ml) } & $\leq 35$ & 32 & 51 & $0.006^{*}$ & 25 & 59 & $0.005^{*}$ \\
\hline & $>35$ & 14 & 5 & & 12 & 6 & \\
\hline \multirow{2}{*}{ Sulfonylurea } & Yes & 19 & 28 & 0.268 & 15 & 32 & 0.371 \\
\hline & No & 27 & 28 & & 22 & 33 & \\
\hline \multirow{2}{*}{ Insulin } & Yes & 18 & 17 & 0.293 & 14 & 21 & 0.486 \\
\hline & No & 28 & 39 & & 23 & 44 & \\
\hline \multirow{2}{*}{ Metformin } & Yes & 7 & 21 & $0.003^{*}$ & 6 & 22 & $0.004^{*}$ \\
\hline & No & 39 & 35 & & 31 & 43 & \\
\hline \multirow{5}{*}{ Site } & Right & 7 & 12 & 0.460 & 6 & 13 & 0.876 \\
\hline & Transverse & 3 & 2 & & 2 & 3 & \\
\hline & Left & 1 & 5 & & 1 & 5 & \\
\hline & Sigmoid & 8 & 11 & & 7 & 12 & \\
\hline & Rectum & 27 & 26 & & 21 & 32 & \\
\hline \multirow{2}{*}{ Adjuvant chemotherapy } & Yes & 25 & 24 & 0.243 & 20 & 29 & 0.321 \\
\hline & No & 21 & 32 & & 17 & 36 & \\
\hline \multirow{2}{*}{ Histologic grade } & I-II & 39 & 46 & 0.992 & 31 & 54 & 0.866 \\
\hline & III & 7 & 10 & & 6 & 11 & \\
\hline \multirow{2}{*}{$\mathrm{T}$} & $\mathrm{T} 1-3$ & 17 & 35 & $0.004^{*}$ & 13 & 39 & $0.010^{*}$ \\
\hline & $\mathrm{T} 4$ & 29 & 21 & & 24 & 26 & \\
\hline \multirow{2}{*}{$\mathrm{N}$} & N0 & 17 & 43 & $<0.001^{*}$ & 14 & 46 & $<0.001^{*}$ \\
\hline & N1-2 & 29 & 13 & & 23 & 19 & \\
\hline \multirow{2}{*}{ E-cadherin } & Positive & 15 & 34 & $0.001^{*}$ & 12 & 37 & $0.003^{*}$ \\
\hline & Negative & 31 & 22 & & 25 & 28 & \\
\hline \multirow{2}{*}{ Vimentin } & Positive & 31 & 24 & $0.019^{*}$ & 26 & 29 & $0.012^{*}$ \\
\hline & Negative & 15 & 32 & & 11 & 36 & \\
\hline
\end{tabular}

${ }^{*} P<0.05$.

mTOR, and Wnt/ $\beta$-catenin [35-38] are involved in EMT occurrence and development, and metformin was observed to act on these pathways [18, 39-41]. Particularly, Zhao et al. discovered that metformin could inhibit IL-6-induced lung cancer EMT by blocking STAT3 phosphorylation [22], Han et al. found the metformin could suppress EMT of thyroid cells through inhibiting mTOR [24], and Banerjee et al. demonstrated that metformin can delay EMT development by interfering with Wnt signaling [41]. Although analogous results had not been reported for CRC, relevant implications and clues were adequately offered from abovementioned studies.

In addition to metformin use, our study revealed that normal presurgery serum CEA and CA19-9 levels, tumor invasion beneath the visceral peritoneum (T1-3), and negative lymph invasion (N0) were associated with positive E-cadherin expression, and high presurgery serum CEA levels, insulin injection, invasion through visceral peritoneum (T4), 
TABLE 4: Multivariate Cox analysis of prognostic factors for DFS and OS.

\begin{tabular}{|c|c|c|c|c|c|c|c|}
\hline \multirow{2}{*}{ Variable } & \multirow{2}{*}{ Category } & \multicolumn{3}{|c|}{ DFS } & \multicolumn{3}{|c|}{ OS } \\
\hline & & $\operatorname{HR}(95 \% \mathrm{CI})$ & $x^{2}$ & $P$ & $\mathrm{HR}(95 \% \mathrm{CI})$ & $\chi^{2}$ & $P$ \\
\hline \multirow{2}{*}{ Age (year) } & $\leq 50$ & $0.898(0.365-2.213)$ & 0.054 & 0.816 & $0.789(0.264-2.359)$ & 0.180 & 0.671 \\
\hline & $>50$ & & & & & & \\
\hline \multirow{2}{*}{ Gender } & Female & $0.547(0.250-1.198)$ & 2.274 & 0.132 & $0.418(0.168-1.040)$ & 3.516 & 0.061 \\
\hline & Male & & & & & & \\
\hline \multirow{2}{*}{ Drinking history } & Yes & $3.451(1.092-10.913)$ & 4.448 & $0.035^{*}$ & $2.578(0.737-9.019)$ & 2.198 & 0.138 \\
\hline & No & & & & & & \\
\hline \multirow{2}{*}{ Smoking history } & Yes & $1.325(0.603-2.912)$ & 0.491 & 0.484 & $2.358(0.875-6.356)$ & 2.873 & 0.090 \\
\hline & No & & & & & & \\
\hline \multirow{2}{*}{ Family history } & Yes & $0.659(0.162-2.671)$ & 0.342 & 0.559 & $0.523(0.106-2.582)$ & 0.634 & 0.426 \\
\hline & No & & & & & & \\
\hline \multirow{2}{*}{ BMI $\left(\mathrm{kg} / \mathrm{m}^{\wedge} 2\right)$} & $\leq 25$ & $0.711(0.336-1.508)$ & 0.790 & 0.673 & $1.457(0.618-3.433)$ & 0.740 & 0.390 \\
\hline & $>25$ & & & & & & \\
\hline \multirow{2}{*}{ CEA (ng/ml) } & $\leq 5$ & $0.857(0.418-1.756)$ & 1.179 & 0.357 & $0.857(0.369-1.994)$ & 0.128 & 0.721 \\
\hline & $>5$ & & & & & & \\
\hline \multirow{2}{*}{ CA19-9 (U/ml) } & $\leq 35$ & $0.694(0.319-1.509)$ & 0.850 & 0.374 & $0.629(0.260-1.520)$ & 1.060 & 0.303 \\
\hline & $>35$ & & & & & & \\
\hline \multirow{2}{*}{ Sulfonylurea } & Yes & $1.629(0.831-3.192)$ & 2.019 & 0.155 & $1.672(0.758-3.690)$ & 1.623 & 0.203 \\
\hline & No & & & & & & \\
\hline \multirow{2}{*}{ Insulin } & Yes & $1.127(0.562-2.261)$ & 0.114 & 0.736 & $1.189(0.545-2.596)$ & 0.189 & 0.664 \\
\hline & No & & & & & & \\
\hline \multirow{2}{*}{ Metformin } & Yes & $3.450(1.273-9.351)$ & 5.924 & $0.015^{*}$ & $3.484(1.036-11.723)$ & 4.066 & $0.044^{*}$ \\
\hline & No & & & & & & \\
\hline \multirow{5}{*}{ Site } & Right & $0.871(0.686-1.106)$ & 1.282 & 0.258 & $0.978(0.744-1.285)$ & 0.026 & 0.871 \\
\hline & Transverse & & & & & & \\
\hline & Left & & & & & & \\
\hline & Sigmoid & & & & & & \\
\hline & Rectum & & & & & & \\
\hline \multirow{2}{*}{ Adjuvant chemotherapy } & Yes & $0.635(0.315-1.280)$ & 1.614 & 0.204 & $0.713(0.312-1.627)$ & 0.647 & 0.421 \\
\hline & No & & & & & & \\
\hline \multirow{2}{*}{ Histologic grade } & I-II & $1.006(0.357-2.839)$ & 0.001 & 0.991 & $1.038(0.332-3.241)$ & 0.004 & 0.949 \\
\hline & III & & & & & & \\
\hline \multirow{2}{*}{$\mathrm{T}$} & $\mathrm{T} 1-3$ & $0.513(0.252-1.046)$ & 3.368 & 0.066 & $0.513(0.227-1.159)$ & 2.577 & 0.108 \\
\hline & $\mathrm{T} 4$ & & & & & & \\
\hline \multirow{2}{*}{$\mathrm{N}$} & N0 & $0.382(0.175-0.834)$ & 5.829 & $0.016^{*}$ & $0.362(0.150-0.872)$ & 5.132 & $0.023^{*}$ \\
\hline & N1-2 & & & & & & \\
\hline \multirow{2}{*}{ E-cadherin } & Positive & $2.252(1.046-4.849)$ & 4.303 & $0.038^{*}$ & $1.923(0.824-4.488)$ & 2.284 & 0.131 \\
\hline & Negative & & & & & & \\
\hline \multirow{2}{*}{ Vimentin } & Positive & $1.409(0.667-2.975)$ & 0.807 & 0.369 & $1.056(0.455-2.451)$ & 0.016 & 0.900 \\
\hline & Negative & & & & & & \\
\hline
\end{tabular}

and positive lymph invasion (N1-2) were associated with positive vimentin expression. A few studies also investigated the relationship between CA19-9 and EMT. For CEA and EMT, in one study, we found that soluble CEA molecules can not only enhance colorectal cell growth but also bind to the TGF$\beta$ receptor, which is a common trigger for EMT and inhibits TGF- $\beta$ signaling [42]. This case was in contrary to our results, but we propose that the inhibition effect was not sufficient to block the mainstream of EMT because there was no further supporting evidence. However, in clinical practice for CRC, abnormally high CEA and CA19-9 levels in serum are more common in patients with advanced disease $[43,44]$. According to previous studies, E-cadherin and vimentin expression was associated with tumor stage, which was in accordance with our results. Yagasaki et al. discovered that E-cadherin and vimentin are associated with axillary metastases in breast cancer [45], and Liu et al. discovered that low E-cadherin expression is correlated with TNM stage for basal-like breast cancer 
[46]. Moreover, E-cadherin and vimentin were also determined to be associated with lymph invasion and TNM stage for lung squamous carcinoma and oral squamous carcinoma $[47,48]$. It should be noted that 3 of the studies mentioned above [45-47] also found the correlation between E-cadherin and vimentin expression and tumor histologic grade; however, such correlation was not significant in our study.

Although patients with normal serum CA19-9 showed significantly longer DFS $(P=0.006)$ and OS $(P=0.005)$ in the Kaplan-Meier analysis, in Cox regression, neither CEA nor CA19-9 was considered as prognostic factors (Table 4), which did not accord with the common views of biomarkers' clinical values. We attribute the results to the following three aspects: (1) limited sample size; (2) low mortality (36.37\%) of local stage (I-III) colorectal cancer in the follow-up period (median: 1678 days); (3) Kaplan-Meier curve and Cox regression were used only for survival data classified by categorical factors or cut-off values. As continuous variables, CEA and CA19-9 were redefined to categorical factors by cut-off values; thus, the original characteristics were inevitably ignored.

The prognostic values of E-cadherin and vimentin have been widely reported in previous studies. E-cadherin and vimentin were found to be prognostic factors for both DFS and OS for lung cancer $[47,49]$ and oral squamous cancer [50]. E-cadherin has also been suggested as a positive indicator for OS for cervical squamous carcinoma [51] and CRC [52]. In our Kaplan-Meier survival analysis, E-cadherin and vimentin expression was demonstrated to be significant for both DFS and OS. However, in further multivariate Cox regression, only E-cadherin was identified as a prognostic factor for DFS. The contradictory results from Cox regression might be attributed to our limited sample size and inclusion criteria, as CRC patients without DM II were excluded from our study. However, the positive result of KaplanMeier analysis could still indicate the prognostic potential of E-cadherin and vimentin.

Patients with metformin not only revealed less EMT in tumors but also showed longer DFS and OS than patients who did not choose metformin for DM II treatment. However, it remains controversial whether metformin could bring survival benefits for cancer patients with DM II. For CRC, our results were supported by several studies [8-12] but do not agree with the results of 2 recent studies $[53,54]$. On the other hand, 6 of 7 studies were retrospective and the remaining study is a meta-analysis [12]. Therefore, the conflicting conclusions need to be verified by a multicenter prospective study with a large cohort.

In summary, combining the result of correlation analysis and survival analysis described above, we suggest that EMT is correlated with CRC progression and can be impeded by metformin. Metformin use and positive E-cadherin expression indicate better prognosis for CRC patients with DM II. Additionally, it is reasonable to believe that the antineoplastic activity of metformin as partially implemented by EMT interference. To further understand the anti-EMT effect of metformin, more inner molecular mechanisms need to be explored in future studies.

\section{Abbreviations}

CRC: Colorectal cancer

EMT: Epithelial-mesenchymal transition

CA19-9: Carbohydrate antigen 19-9

CEA: Carcinoembryonic antigen

AMPK: Adenosine monophosphate-activated protein kinase

TGF- $\beta$ : Transforming growth factor beta

JAK: Janus kinase

STAT: Signal transducer and activator of transcription

mTOR: Mammalian target of rapamycin

DFS: Disease-free survival

OS: Overall survival.

\section{Conflicts of Interest}

The authors declare that they have no conflict of interests.

\section{Acknowledgments}

This study was supported by Natural Science Foundation of Shandong Province (Z2005Co2) and Science and Technology Development Project of Shandong Province (2014GSF118052).

\section{References}

[1] J. Ferlay, I. Soerjomataram, R. Dikshit et al., "Cancer incidence and mortality worldwide: sources, methods and major patterns in GLOBOCAN 2012," International Journal of Cancer, vol. 136, no. 5, pp. E359-E386, 2015.

[2] S. C. Larsson, N. Orsini, and A. Wolk, "Diabetes mellitus and risk of colorectal cancer: a meta-analysis," Journal of the National Cancer Institute, vol. 97, no. 22, pp. 1679-1687, 2005.

[3] D. Leroith, R. Novosyadlyy, E. J. Gallagher, D. Lann, A. Vijayakumar, and S. Yakar, "Obesity and type 2 diabetes are associated with an increased risk of developing cancer and a worse prognosis; epidemiological and mechanistic evidence," Experimental and Clinical Endocrinology \& Diabetes, vol. 116, Supplement 1, pp. S4-S6, 2008.

[4] K. B. Stein, C. F. Snyder, B. B. Barone et al., "Colorectal cancer outcomes, recurrence, and complications in persons with and without diabetes mellitus: a systematic review and meta-analysis," Digestive Diseases \& Sciences, vol. 55, no. 7, pp. 1839$1851,2010$.

[5] K. T. Mills, C. F. Bellows, A. E. Hoffman, T. N. Kelly, and G. Gagliardi, "Diabetes mellitus and colorectal cancer prognosis: a meta-analysis," Diseases of the Colon \& Rectum, vol. 56, no. 11, pp. 1304-1319, 2013.

[6] J. M. Evans, L. A. Donnelly, A. M. Emslie-Smith, D. R. Alessi, and A. D. Morris, "Metformin and reduced risk of cancer in diabetic patients," BMJ, vol. 330, no. 7503, pp. 1304-1305, 2005.

[7] G. Libby, L. A. Donnelly, P. T. Donnan, D. R. Alessi, A. D. Morris, and J. M. Evans, "New users of metformin are at low risk of incident cancer: a cohort study among people with type 2 diabetes," Diabetes Care, vol. 32, no. 9, pp. 1620-1625, 2009.

[8] J. H. Lee, T. I. Kim, S. M. Jeon, S. P. Hong, J. H. Cheon, and W. H. Kim, "The effects of metformin on the survival of colorectal 
cancer patients with diabetes mellitus," International Journal of Cancer, vol. 131, no. 3, pp. 752-759, 2012.

[9] C. R. Garrett, H. M. Hassabo, N. A. Bhadkamkar et al., "Survival advantage observed with the use of metformin in patients with type II diabetes and colorectal cancer," British Journal of Cancer, vol. 106, no. 8, pp. 1374-1378, 2012.

[10] S. Spillane, K. Bennett, L. Sharp, and T. I. Barron, “A cohort study of metformin exposure and survival in patients with stage I-III colorectal cancer," Cancer Epidemiology, Biomarkers \& Prevention, vol. 22, no. 8, pp. 1364-1373, 2013.

[11] H. D. Skinner, C. H. Crane, C. R. Garrett et al., "Metformin use and improved response to therapy in rectal cancer," Cancer Medicine, vol. 2, no. 1, pp. 99-107, 2013.

[12] X. K. He, T. T. Su, J. M. Si, and L. M. Sun, "Metformin is associated with slightly reduced risk of colorectal cancer and moderate survival benefits in diabetes mellitus: a meta-analysis," Medicine, vol. 95, no. 7, 2016.

[13] A. P. Morel, M. Lièvre, C. Thomas, G. Hinkal, S. Ansieau, and A. Puisieux, "Generation of breast cancer stem cells through epithelial-mesenchymal transition," PLoS One, vol. 3, no. 8, article e2888, 2008.

[14] A. J. Armstrong, M. S. Marengo, S. Oltean et al., "Circulating tumor cells from patients with advanced prostate and breast cancer display both epithelial and mesenchymal markers," Molecular Cancer Research, vol. 9, no. 8, pp. 997-1007, 2011.

[15] A. Singh and J. Settleman, "EMT, cancer stem cells and drug resistance: an emerging axis of evil in the war on cancer," Oncogene, vol. 29, no. 34, pp. 4741-4751, 2010.

[16] C. L. Chaffer and R. A. Weinberg, "A perspective on cancer cell metastasis," Science, vol. 331, no. 6024, pp. 1559-1564, 2011.

[17] A. Vazquezmartin, C. Oliverasferraros, S. Cufí, S. Del Barco, B. Martin-Castillo, and J. A. Menendez, "Metformin regulates breast cancer stem cello ntogeny by transcriptional regulation of the epithelial-mesenchymal transition (EMT) status," Cell Cycle, vol. 9, no. 18, pp. 3807-3814, 2010.

[18] S. Cufí, A. Vazquez-Martin, C. Oliveras-Ferraros, B. MartinCastillo, J. Joven, and J. A. Menendez, "Metformin against TGF $\beta$-induced epithelial-to-mesenchymal transition (EMT): from cancer stem cells to aging-associated fibrosis," Cell Cycle, vol. 9, no. 22, pp. 4461-4468, 2010.

[19] M. Cerezo, M. Tichet, P. Abbe et al., "Metformin blocks melanoma invasion and metastasis development in AMPK/p53dependent manner," Molecular Cancer Therapeutics, vol. 12, no. 8, pp. 1605-1615, 2013.

[20] C. Qu, W. Zhang, G. Zheng, Z. Zhang, J. Yin, and Z. He, "Metformin reverses multidrug resistance and epithelial-mesenchymal transition (EMT) via activating AMPactivated protein kinase (AMPK) in human breast cancer cells," Molecular and Cellular Biochemistry, vol. 386, no. 2, pp. 63-71, 2014.

[21] Y. Wang, B. Yao, Y. Wang et al., "Increased FoxM1 expression is a target for metformin in the suppression of EMT in prostate cancer," International Journal of Molecular Medicine, vol. 33, no. 6, pp. 1514-1522, 2014.

[22] Z. Zhao, X. Cheng, Y. Wang et al., "Metformin inhibits the IL6-induced epithelial-mesenchymal transition and lung adenocarcinoma growth and metastasis," PLoS One, vol. 9, no. 4, article e95884, 2014.

[23] J. Zhang, C. Shen, L. Wang et al., "Metformin inhibits epithelial-mesenchymal transition in prostate cancer cells: involvement of the tumor suppressor miR30a and its target gene
SOX4," Biochemical \& Biophysical Research Communications, vol. 452, no. 3, pp. 746-752, 2014.

[24] B. Han, H. Cui, L. Kang et al., "Metformin inhibits thyroid cancer cell growth, migration, and EMT through the mTOR pathway," Tumour Biology, vol. 36, no. 8, pp. 6295-6304, 2015.

[25] M. Rønning, H. S. Blix, B. T. Harbø, and H. Strøm, “Different versions of the anatomical therapeutic chemical classification system and the defined daily dose - are drug utilisation data comparable?" European Journal of Clinical Pharmacology, vol. 56, no. 9-10, pp. 723-727, 2000.

[26] K. Polyak and R. A. Weinberg, "Transitions between epithelial and mesenchymal states: acquisition of malignant and stem cell traits," Nature Reviews Cancer, vol. 9, no. 4, pp. 265-273, 2009.

[27] M. Leader, M. Collins, J. Patel, and K. Henry, "Vimentin: an evaluation of its role as a tumour marker," Histopathology, vol. 11, no. 1, pp. 63-72, 1987.

[28] G. Rena, E. R. Pearson, and K. Sakamoto, "Molecular mechanism of action of metformin: old or new insights?" Diabetologia, vol. 56, no. 9, pp. 1898-1906, 2013.

[29] M. C. Towler and D. G. Hardie, "AMP-activated protein kinase in metabolic control and insulin signaling," Circulation Research, vol. 100, no. 3, pp. 328-341, 2007.

[30] J. A. Menendez, "Metformin and cancer: doses, mechanisms and the dandelion and hormetic phenomena," Cell Cycle, vol. 9, no. 6, pp. 1057-1064, 2010.

[31] Y. K. Choi and K. G. Park, "Metabolic roles of AMPK and metformin in cancer cells," Molecules and Cells, vol. 36, no. 4, pp. 279-287, 2013.

[32] M. Zakikhani, R. J. Dowling, N. Sonenberg, and M. N. Pollak, "The effects of adiponectin and metformin on prostate and colon neoplasia involve activation of AMP-activated protein kinase," Cancer Prevention Research, vol. 1, no. 5, pp. 369-375, 2008.

[33] M. Hyun-Seuk and C. S. Mantzoros, "Adiponectin and metformin additively attenuate IL $1 \beta$-induced malignant potential of colon cancer," Endocrine Related Cancer, vol. 20, no. 6, pp. 849-859, 2013.

[34] S. Thakur, S. Viswanadhapalli, J. B. Kopp et al., "Activation of AMP-activated protein kinase prevents TGF- $\beta 1$-induced epithelial-mesenchymal transition and myofibroblast activation," American Journal of Pathology, vol. 185, no. 8, pp. 2168-2180, 2015.

[35] J. Fuxe, T. Vincent, and A. Garcia de Herreros, "Transcriptional crosstalk between TGF- $\beta$ and stem cell pathways in tumor cell invasion: role of EMT promoting Smad complexes," Cell Cycle, vol. 9, no. 12, pp. 2363-2374, 2010.

[36] H. Xiong, J. Hong, W. Du et al., "Roles of STAT3 and ZEB1 proteins in E-cadherin down-regulation and human colorectal cancer epithelial-mesenchymal transition," Journal of Biological Chemistry, vol. 287, no. 8, pp. 5819-5832, 2012.

[37] P. Gulhati, K. A. Bowen, J. Liu et al., "mTORC1 and mTORC2 regulate EMT, motility, and metastasis of colorectal cancer via RhoA and Racl signaling pathways," Cancer Research, vol. 71, no. 9, pp. 3246-3256, 2011.

[38] Y. Yanaka, T. Muramatsu, H. Uetake, K. Kozaki, and J. Inazawa, "miR-544a induces epithelial-mesenchymal transition through the activation of WNT signaling pathway in gastric cancer," Carcinogenesis, vol. 36, no. 11, pp. 13631371, 2015. 
[39] H. A. Hirsch, D. Iliopoulos, and K. Struhl, "Metformin inhibits the inflammatory response associated with cellular transformation and cancer stem cell growth," Proceedings of the National Academy of Sciences of the United States of America, vol. 110, no. 3, pp. 972-977, 2013.

[40] A. Kalender, A. Selvaraj, S. Y. Kim et al., "Metformin, independent of AMPK, inhibits mTORC1 in a Rag GTPase-dependent manner," Cell Metabolism, vol. 11, no. 5, pp. 390-401, 2010.

[41] P. Banerjee, S. Dutta, and R. Pal, "Dysregulation of Wntsignaling and a candidate set of miRNAs underlie the effect of metformin on neural crest cell development," Stem Cells, vol. 34, no. 2, pp. 334-345, 2015.

[42] Y. Li, H. Cao, Z. Jiao et al., "Carcinoembryonic antigen interacts with TGF- $\beta$ receptor and inhibits TGF- $\beta$ signaling in colorectal cancers," Cancer Research, vol. 70, no. 20, pp. 8159-8168, 2010.

[43] C. G. Moertel, J. R. O’Fallon, V. L. Go, M. J. O’Connell, and G. S. Thynne, "The preoperative carcinoembryonic antigen test in the diagnosis, staging, and prognosis of colorectal cancer," Cancer, vol. 58, no. 3, pp. 603-610, 1986.

[44] M. Plebani, M. De Paoli, D. Basso et al., "Serum tumor markers in colorectal cancer staging, grading, and follow-up," Journal of Surgical Oncology, vol. 62, no. 4, pp. 239-244, 1996.

[45] R. Yagasaki, M. Noguchi, M. Minami, and M. Earashi, "Clinical significance of E-cadherin and vimentin co-expression in breast cancer," International Journal of Oncology, vol. 9, no. 4, pp. 755-761, 1996.

[46] T. Liu, X. Zhang, M. Shang et al., "Dysregulated expression of Slug, vimentin, and E-cadherin correlates with poor clinical outcome in patients with basal-like breast cancer," Journal of Surgical Oncology, vol. 107, no. 2, pp. 188-194, 2013.

[47] H. Zhang, J. Liu, D. Yue et al., "Clinical significance of E-cadherin, $\beta$-catenin, vimentin and S100A4 expression in completely resected squamous cell lung carcinoma," Journal of Clinical Pathology, vol. 66, no. 11, pp. 937-945, 2013.

[48] J. Zhou, D. Tao, Q. Xu, Z. Gao, and D. Tang, "Expression of Ecadherin and vimentin in oral squamous cell carcinoma," International Journal of Clinical \& Experimental Pathology, vol. 8, no. 3, pp. 3150-3154, 2014.

[49] F. Richardson, G. D. Young, R. Sennello et al., "The evaluation of E-cadherin and vimentin as biomarkers of clinical outcomes among patients with non-small cell lung cancer treated with erlotinib as second- or third-line therapy," Anticancer Research, vol. 32, no. 2, pp. 537-552, 2012.

[50] L. K. Liu, X. Y. Jiang, X. X. Zhou, D. M. Wang, X. L. Song, and H. B. Jiang, "Upregulation of vimentin and aberrant expression of E-cadherin/ $\beta$-catenin complex in oral squamous cell carcinomas: correlation with the clinicopathological features and patient outcome," Modern Pathology, vol. 23, no. 2, pp. 213-224, 2010.

[51] Y. Cheng, Y. Zhou, W. Jiang et al., "Significance of E-cadherin, $\beta$-catenin, and vimentin expression as postoperative prognosis indicators in cervical squamous cell carcinoma," Human Pathology, vol. 43, no. 8, pp. 1213-1220, 2012.

[52] X. He, Z. Chen, M. Jia, and X. Zhao, "Downregulated Ecadherin expression indicates worse prognosis in Asian patients with colorectal cancer: evidence from meta-analysis," PLoS One, vol. 8, no. 7, article e70858, 2013.
[53] M. M. Zanders, M. P. van Herk-Sukel, P. A. Vissers, R. M. Herings, H. R. Haak, and L. V. van de Poll-Franse, "Are metformin, statin and aspirin use still associated with overall mortality among colorectal cancer patients with diabetes if adjusted for one another?" British Journal of Cancer, vol. 113, no. 3, pp. 403-410, 2015.

[54] Ú. C. Mc Menamin, L. J. Murray, C. M. Hughes, and C. R. Cardwell, "Metformin use and survival after colorectal cancer: a population-based cohort study," International Journal of Cancer, vol. 138, no. 2, pp. 369-379, 2015. 


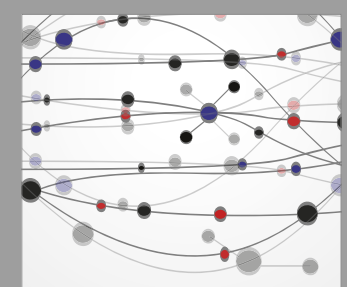

The Scientific World Journal
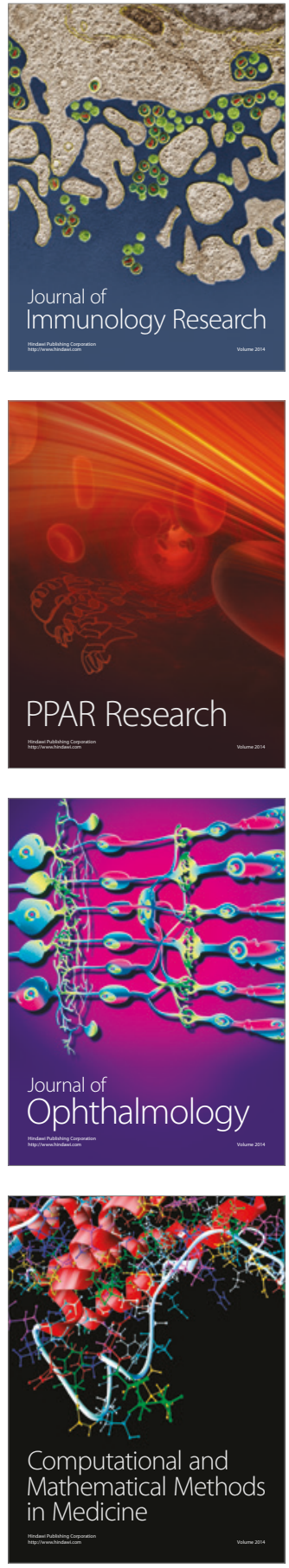

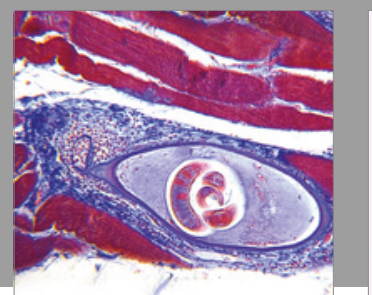

Gastroenterology Research and Practice
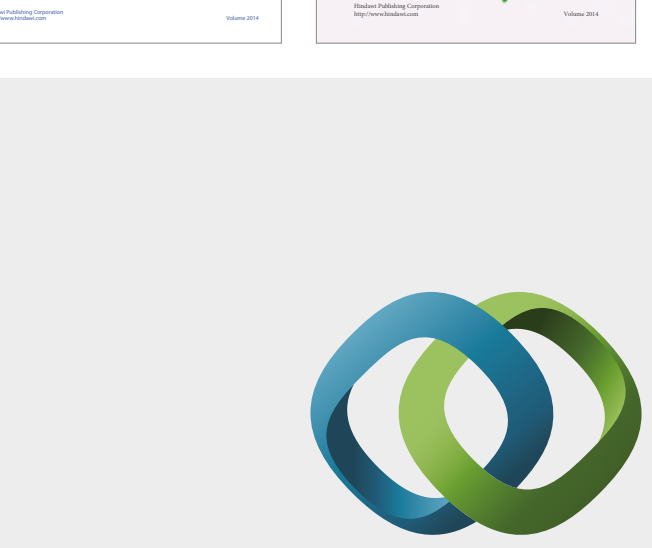

\section{Hindawi}

Submit your manuscripts at

https://www.hindawi.com
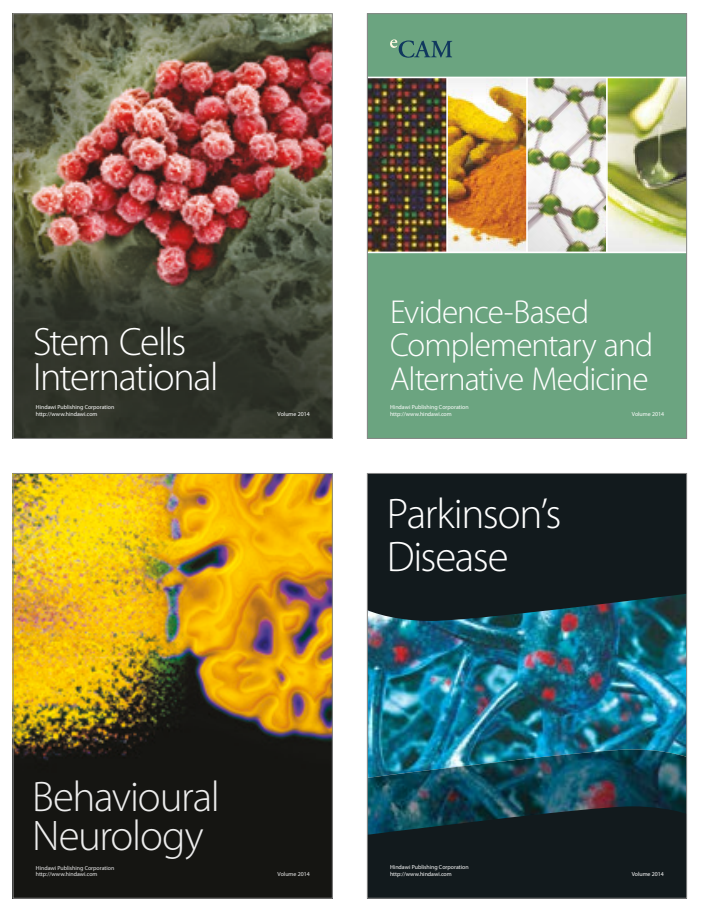
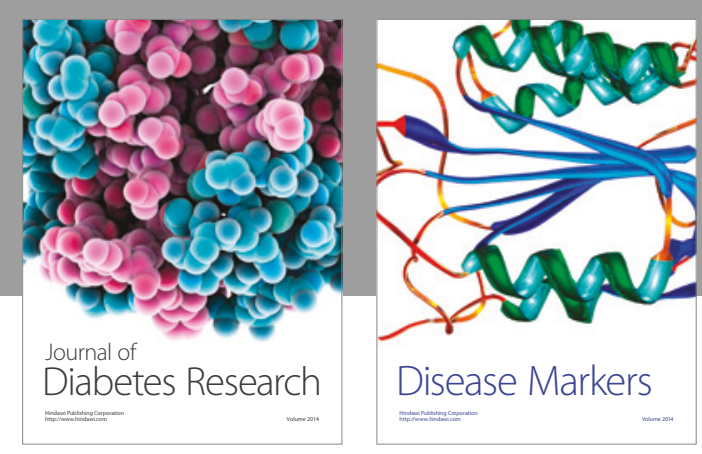

Disease Markers
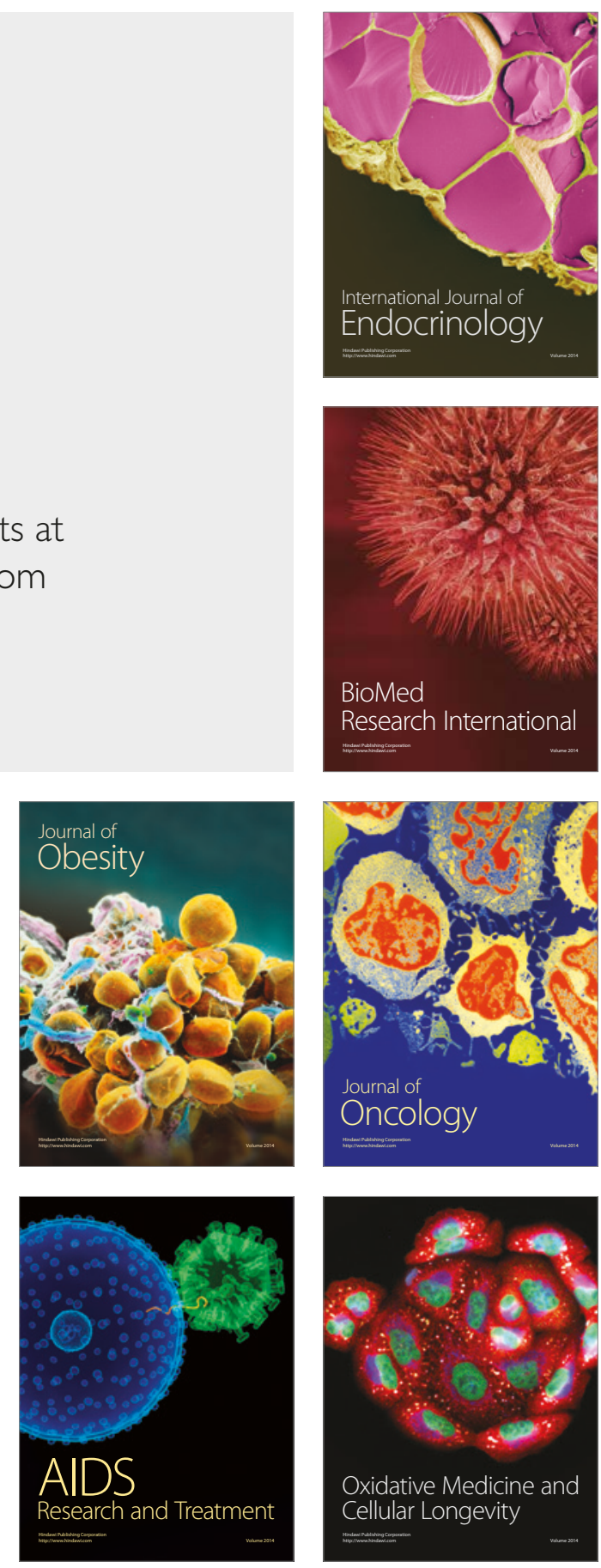